\title{
THE EQUIVALENCE OF TWO EXTENSIONS OF LEBESGUE AREA
}

EDWARD SILVERMAN

The area of a triangle in a Banach space was defined in [5] and then Lebesgue's definition for the area of a continuous function (surface) was applicable to a surface in a Banach space. If $X$ is a continuous function on a closed simply-connected Jordan region $J$ into a Banach space $B$ let $L_{B}(X)$ denote its area in accordance with this definition. Let $m$ be the space of bounded sequences [1]. It was shown that isometric functions ${ }^{1}$ on $J$ into $m$ have the same $L_{m}$ area. If $X$ is continuous on $J$ into a metric space $M$ there is a continuous function $x$ on $J$ into $m$ which is isometric with $X$. Define $L(X)$ to be equal to $L_{m}(x)$.

If $X$ is continuous on $J$ into a Euclidean space $E$, then $L_{E}(X)$ is the classical Lebesgue area of $X$. It was shown that $L(X)=L_{E}(X)$ and hence it seemed reasonable to call $L$ an extension of Lebesgue area which applied to surfaces in a metric space.

Now let us consider those functions which are continuous on $J$ into a Banach space $B$. There are two Lebesgue type areas available, $L$ and $L_{B}$. Morrey's representation theorem [3] and the cyclic additivity theory were used to show that $L=L_{B}$ under a variety of conditions, in particular, as mentioned above, if $B=E$.

The hypothesis in Morrey's theorem that the functions range in $E$ was too strong a restriction to enable us to conclude that $L(X)$ $=L_{B}(X)$ for all Banach spaces $B$ and continuous functions $X$ on $J$ into $B$. In [6] Morrey's theorem is extended to apply to surfaces in a metric space. We shall see that this version of Morrey's theorem can be used to show that $L$ and $L_{B}$ are equivalent, whenever $L_{B}$ is applicable.

Let $J$ be contained in the $u, v$ plane. According to Cesari [2], a function $X$ on $J$ into $E$ is a $D$-mapping if each component of $X$ is A.C.T. in $J^{0}$, the interior of $J$, and if all of the partial derivatives are square summable over $J^{0}$. This definition can be generalized so as to apply to functions with range in a Banach space [6]. The property of being a $D$-mapping is invariant under an isometric transformation. If $X$ is a $D$-mapping in $B$ then $X$ is of class $\bar{L}_{B}$ in the sense of [5] (see [4, IV. 4.33]).

Received by the editors April 7, 1958.

1 If $X$ and $Y$ map $J$ into metric spaces $M$ and $N$ respectively, then $X$ and $Y$ are isometric if $\operatorname{dist}_{M}(X(u), X(v))=\operatorname{dist}_{N}(Y(u), Y(v))$ for all $u, v \in J$. 
The equivalence of $L$ and $L_{B}$, whenever the latter is defined, is a result of the cyclic additivity theory, the fact that Fréchet equivalent functions have the same Lebesgue area, $L$ or $L_{B}$, and Theorems 1 and 2 (from [5] and [6], respectively).

THEOREM 1. If range $X \subset B$ then $L(X) \leqq L_{B}(X)$. If $X$ is of class $\bar{L}_{B}$ then $L(X)=L_{B}(X)$.

THEOREM 2. If range $x \subset m$, if $x$ is light, and if $L(x)<+\infty$, then there is a $D$-mapping $y$ which is Fréchet equivalent to $x$.

Theorem 3. If $X$ is continuous on $J$ into $B$ then $L(X)=L_{B}(X)$.

Proof. We can use Theorem 1 and the cyclic additivity theory to assume, without loss of generality, that $X$ is light and that $L(X)<+\infty$. Take $x$ isometric with $X$, range $x \subset m$. According to Theorem 2 there is a $D$-mapping $y$ which is Fréchet equivalent to $x$. For each $u \in J$ choose $v$ to satisfy $x(v)=y(u)$ and define $Y(u)=X(v)$. (If $v_{1}$ and $v_{2}$ correspond to $u$ then $x\left(v_{1}\right)=x\left(v_{2}\right)$ which implies that $X\left(v_{1}\right)=X\left(v_{2}\right)$.) It is easy to see that $X$ and $Y$ are Fréchet equivalent and that $Y$ and $y$ are isometric. Thus $Y$ is a $D$-mapping and Theorem 1 enables us to conclude that $L_{B}(X)=L_{B}(Y)=L(Y)=L(X)$.

\section{REFERENCES}

1. S. Banach, Theorie des operations lineaires, Warsaw, 1932.

2. L. Cesari, Surface area, Princeton University Press, 1956.

3. C. B. Morrey, A class of representations of manifolds, Amer. J. Math. vol. 55 (1933) pp. 683-707.

4. T. Rad6, Length and area, Amer. Math. Soc. Colloquium Publications, vol. 30 , 1948.

5. E. Silverman, Definitions of Lebesgue area for surfaces in metric spaces, Riv. Mat. Univ. Parma vol. 2 (1951) pp. 47-76.

6. - Morrey's representation theorem for surfaces in metric spaces, Pacific J. Math. vol. 7 (1957) pp. 1677-1690.

Purdue University 